15

\title{
Использование атомных пучков средних энергий для твердотельной PIXE-диагностики
}

\author{
() И.Т. Серенков, В.И. Сахаров
}

Физико-технический институт им. А.Ф. Иофрфе РАН, 194021 Санкт-Петербург, Россия

e-mail: r.ilin@mail.ioffe.ru

Поступило в Редакцию 17 июня 2020 г.

В окончательной редакции 14 июля 2020 г.

Принято к публикации 6 августа 2020 г.

Рассмотрены возможности использования ионных пучков средних (сотни $\mathrm{keV}$ ) энергий для диагностики твердого тела методом измерения спектров характеристического рентгеновского излучения. Предложена модификация метода, состоящая в применении пучков нейтральных атомов в качестве зондирующих. Показано, что переход на такие пучки позволяет исключить негативное влияние эффектов, связанных с возникновением поверхностного потенциала, обусловленного накоплением заряда, при работе с диэлектрическими образцами.

Ключевые слова: характеристическое рентгеновское излучение, диагностика твердого тела, ионные пучки, средние энергии.

DOI: 10.21883/JTF.2021.01.50290.206-20

\section{Введение}

Регистрация характеристического рентгеновского излучения, возникающего при облучении образца пучком ионов (далее - PIXE, Particle Induced X-Ray Emission) как диагностический метод активно развивается с 70-х годов прошлого века и используется для изучения как твердого тела, так и жидкостей, биологических (в том числе пищевых) объектов, археологических находок и т. п. [1]. Метод РІХЕ позволяет установить качественный элементный состав исследуемого образца, проследить наличие примесей, а также сделать количественную оценку содержания тех или иных элементов в составе исследуемого объекта. К недостаткам PIXE, как метода твердотельной диагностики (в настоящей работе будем рассматривать только такое приложение), можно отнести отсутствие глубинного разрешения, а также при построении расчетных спектров с целью сравнения с экспериментальными необходимость привлечения большого количества сторонних баз данных по торможению частиц пучка в материале объекта, сечениям ионизации внутренних оболочек, квантовым выходам, коэффициентам Костера-Кронига, относительной интенсивности линий, коэффициентам поглощения квантов в исследуемой среде. Хотя эти данные имеются в литературе, они в основном являются результатами расчетов, выполненных в рамках различных моделей и приближений, что затрудняет установление абсолютных количественных характеристик состава образца. В то же время метод PIXE может быть эффективно использован в ситуациях, когда требуется провести сравнение изучаемого объекта с другим, например, эталонным. Если сравнивать PIXE с другой распространенной пучковой диагностикой, а именно с RBS (Rutherford Backscattering Spectrometry), то можно отметить существенно более высокую чувствительность PIXE к легким элементам в относительно тяжелой матрице. Отметим также, что при PIXE-исследовании кристаллов в сочетании с каналированием ионов метод позволяет путем измерения зависимостей интенсивностей различных линий от угла между осью пучка частиц и осью кристалла (так называемым „сканам“) сделать выводы об особенностях кристаллических подрешеток отдельных элементов в сложных кристаллах [2].

Как отмечают многие исследователи, использующие PIXE-диагностику, главным негативным фактором, затрудняющим анализ спектров, является наличие фона тормозного излучения (ТИ, bremmstrahlung) [3]. Выражается это в том, что линии характеристического излучения оказываются поднятыми на энергозависимый „Пьедестал“, причем интенсивность этого непрерывного по частоте квантов излучения зачастую превышает интенсивности линий, особенно при выявлении относительно небольших примесей в исследуемом веществе, что ухудшает отношение сигнал/шум, снижая чувствительность. Следуя классификации, предложенной K. Ishii с соавторами [4], отметим, что основными физическими явлениями, обусловливающими фон ТИ, являются, во-первых, „атомное ТИ“, во-вторых, „вторичноэлектронное ТИ““. Первое связано с излучением при возбуждении атома налетающей частицей с последующим излучением рентгеновского кванта, второе - с потерей энергии на излучение выбитым из атома электроном при его движении в веществе, причем с повышением энергии частиц пучка от сотен $\mathrm{keV}$ до единиц $\mathrm{MeV}$ второй канал начинает доминировать. Если же исследуемый образец является хорошим диэлектриком, то вследствие зарядки поверхности ионами пучка добавляется сигнал, вызванный проникновением в образец ускоренных электронов 
из окружающего пространства, что приводит не только к увеличению фона ТИ, но и к наложению на спектр линий характеристического излучения, обусловленного ускоренными на потенциале поверхности электронами. Такие линии, обладая своим распределением интенсивностей, отличным от распределения в спектре PIXE, порожденном только протонной ионизацией внутренних оболочек атомов, затрудняют PIXE-анализ образца.

Таким образом, важной, на наш взгляд, задачей является рассмотрение экспериментальных условий, снижающих, вплоть до полной ликвидации, вышеуказанные негативные явления. В настоящей работе предлагаются некоторые способы решения данной проблемы.

Кратко опишем нашу экспериментальную установку. Ионы, извлеченные из ВЧ-источника, ускорялись линейной системой до (максимально) $230 \mathrm{keV}$, селектировались по отношению заряда к массе магнитным массмонохроматором, далее проходили камеры нейтрализации и очистки от заряженной фракции (см. разд. 3) и поступали в измерительную камеру, где располагались рентгеновские детекторы и устанавливался исследуемый образец с возможностью его качания в двух взаимоперпендикулярных плоскостях, что необходимо для измерений в режиме каналирования. Давление в измерительной камере составляло $2 \cdot 10^{-7}-5 \cdot 10^{-8}$ Torr. Площадка, к которой крепился образец, была электрически изолирована от заземленных конструкций и соединялась с „землей“ через цепь, включавшую токовый цифровой измерительный прибор и изолированный аккумуляторный источник напряжения на $\sim 40 \mathrm{~V}$ (,плюсом“ к образцу), служивший для подавления ион-электронной эмиссии. Ток ионного пучка, падающего на образец, составлял 2-3 nA, характерная экспозиция при измерениях на ионном пучке составляла 20-40 min, на атомном (см. разд. 3$)-2-3 \mathrm{~h}$.

\section{1. Использование ионных пучков средних энергий}

Традиционно в PIXE используются пучки протонов, реже - ионов $\mathrm{He}^{+}$, с энергиями порядка нескольких, обычно $1.5-3, \mathrm{MeV}$, что связано с тем, что эти энергии соответствуют области максимальных значений сечений ионизации внутренних оболочек атомов. Переход на пучки средних (сотни keV) энергий снижает указанные сечения и соответственно интенсивность излучения на 2-3 порядка, кроме того, сужаются как диапазон просматриваемых глубин образца, так и диапазон энергий надежно регистрируемых квантов. В то же время PIXE-измерения на средних энергиях обладают рядом преимуществ по отношению к традиционной $\mathrm{MeV}$-области. Кратко перечислим основные достоинства таких измерений.

1. Не требуется использование громоздких MeV-ycкорителей. Например, авторами работы [5] создан компактный калибровочный источник монохроматического

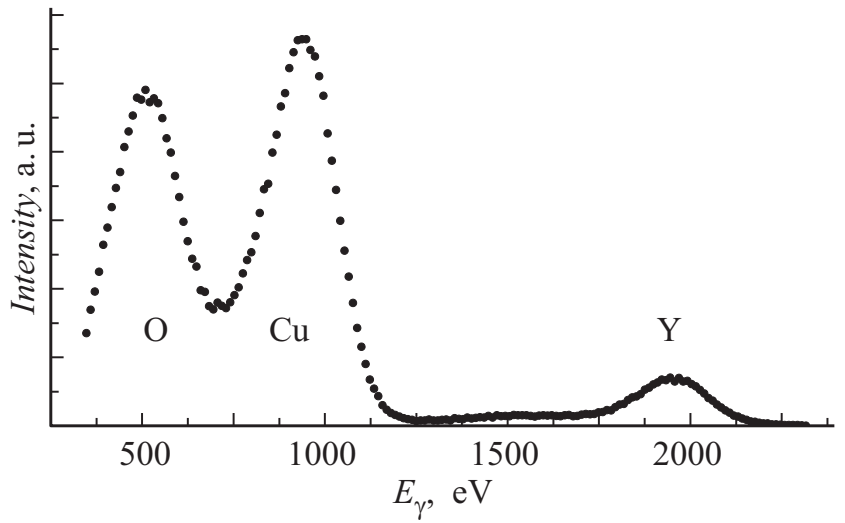

Рис. 1. РIXE-спектр монокристалла $\mathrm{YBa}_{2} \mathrm{Cu}_{3} \mathrm{O}_{7-x}$, полученный на пучке протонов с энергией $230 \mathrm{keV}$.

рентгеновского излучения разных частот на базе ионного ускорителя с энергией $165 \mathrm{keV}$.

2. Возможность исследования сравнительно тонких (до $1 \mu \mathrm{m}$ ) слоев и пленок с полным либо существенным подавлением линий материала подложки. Более того, как отмечается в [6], появляется возможность, используя большие по отношению к $\mathrm{MeV}$-области, значения сечений торможения ионов, путем варьирования энергии пучка получать данные о глубинном распределении примесей.

3. Возможность снижения толщины фильтра, устанавливаемого перед детектором для защиты последнего от попадания в него обратнорассеянных ионов. Обычно применяются фильтры из бериллия или лавсановой (mylar) пленки. Если в детекторах, предназначенных для PIXE-измерений на MeV-ионах, характерное значение толщины фильтра составляет 20-50 $\mu \mathrm{m}$, то с переходом в диапазон энергий сотни $\mathrm{keV}$ достаточно толщины в 2-4 $\mu \mathrm{m}$. Снижение толщины фильтра, в свою очередь, позволяет за счет уменьшения поглощения уверенно регистрировать характеристическое излучение с энергией ниже $1 \mathrm{keV}$, например, $K$-линию кислорода $(525 \mathrm{keV})$. На рис. 1 приведен измеренный нами с помощью разработанного в Радиевом институте им. В.Г. Хлопина детектора из особого чистого кремния, охлаждаемого жидким азотом, PIXE-спектр кристалла высокотемпературной сверхпроводящей (ВТСП) керамики $\mathrm{YBa}_{2} \mathrm{Cu}_{3} \mathrm{O}_{7-x}$, полученный с применением пучка протонов с энергией $230 \mathrm{keV}$; толщина Ве фильтра перед детектором составляла $\sim 3 \mu \mathrm{m}$.

4. Переход на средние энергии при измерении сканов (см. Введение) снижает требования к угловому разрешению аппаратуры, поскольку угловая ширина скана $\propto E^{-1 / 2}$ [7]. На рис. 2 представлены измеренные нами сканы ВТСП монокристалла и пленки толщиной $\sim 220 \mathrm{~nm}$ того же состава, что на рис. 1 , демонстрирующие различие структурной упорядоченности различных элементов. Пленка осаждена на подложку $\mathrm{MgO}$. Как показали расчеты и контрольные измерения, для пленки 

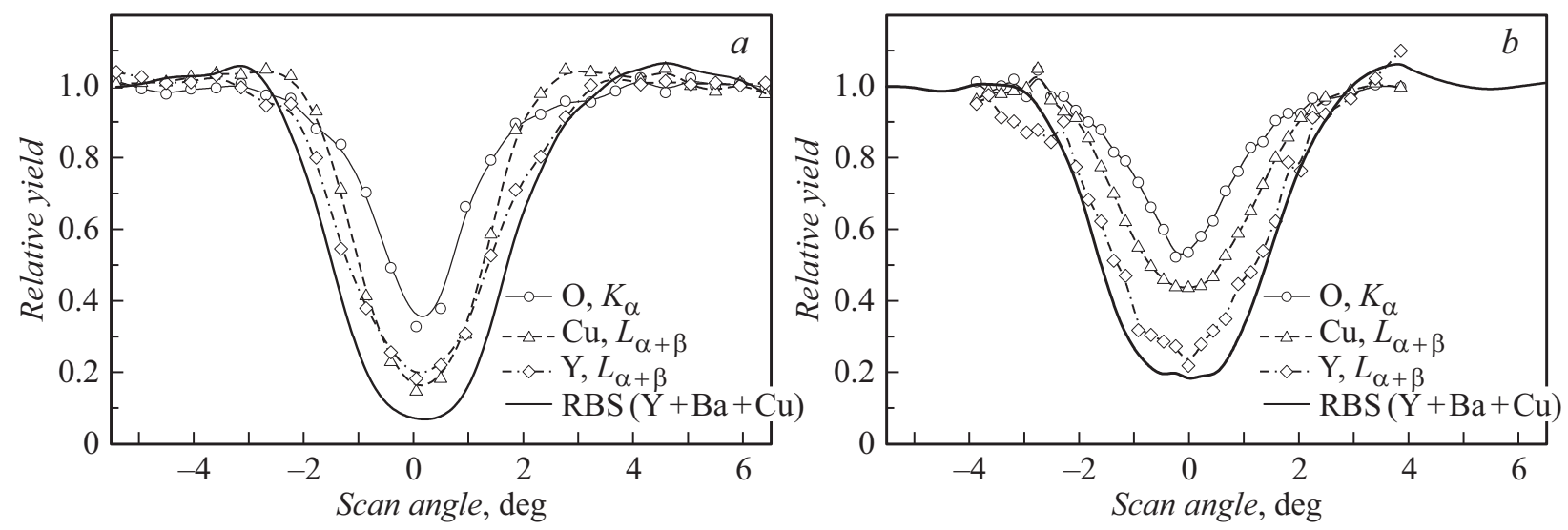

Рис. 2. PIXE- и RBS-угловые сканы монокристалла (слева) и пленки (справа) ВТСП-керамики, полученные на пучке протонов с энергией $230 \mathrm{keV}$.

вклад сигнала кислорода подложки составляет не более $10 \%$ суммарного сигнала кислорода.

Рисунок показывает ширину РІХЕ-сканов для металлов $\sim 3^{\circ}$. При повышении энергии в 10 раз, т.е. при переходе в $\mathrm{MeV}$-диапазон, эта величина станет меньше $1^{\circ}$, что предъявляет повышенные требования к расходимости зондирующего пучка. Отметим, правда, что при измерении сканов в $\mathrm{MeV}$-диапазоне иногда применяются пучки $\mathrm{He}^{+}$. Так, в работе [8] при PIXEисследованиях аналогичных структур на пучке $\mathrm{He}^{+}$, $2.0 \mathrm{MeV}$, измеренная ширина скана составила $\sim 2^{\circ}$.

5. Использование пучков средних энергий позволяет практически полностью избавиться от излучения, связанного с движением электронов в образце. Подробно это будет рассмотрено в следующем разделе.

\section{2. Фон тормозного излучения в спектрах PIXE при использовании ионов средних энергий}

Как показывают наши измерения PIXE-спектров различных проводящих образцов на протонах с энергией $230 \mathrm{keV}$, фон тормозного излучения в таких ситуациях практически необнаружим. Под „проводящими“ мы будем понимать такие кристаллы и системы пленка+подложка, проводимость которых обеспечивает либо отсутствие на поверхности накопления заряда, индуцированного ионами падающего пучка, либо значение потенциала, связанного с этим зарядом, не превышающее $\sim 1 \mathrm{kV}$. На рис. 3 (см. также рис. 1) показан РІХЕ-спектр пленки $\mathrm{BaSn}_{x} \mathrm{Ti}_{1-x} \mathrm{O}_{3}$ на проводящей подложке $\mathrm{SiC}$. Этот и последующие PIXE-спектры измерялись с помощью серийного детектора XR-100CR фирмы AMPTEK c бериллиевым фильтром толщиной $\sim 13 \mu \mathrm{m}$, так что кислород в данном случае не регистрировался.

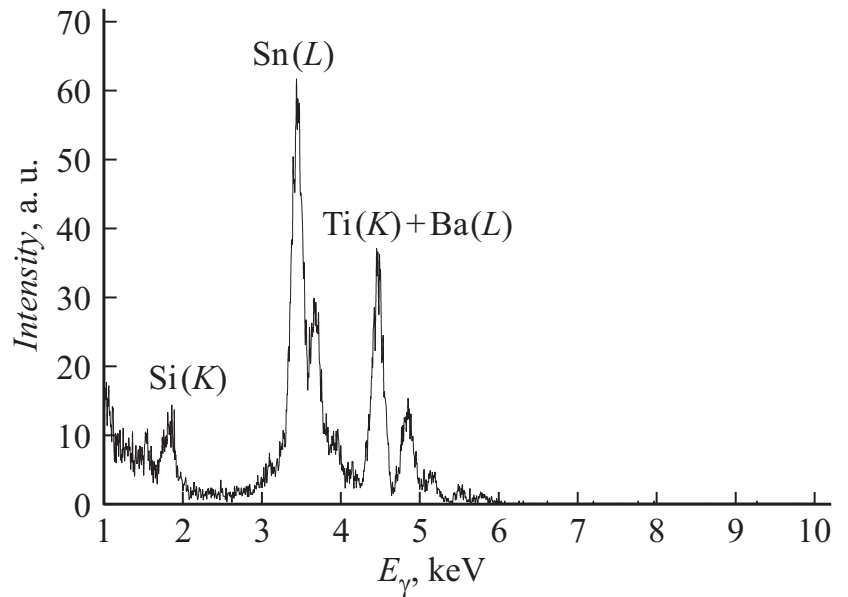

Pис. 3. PIXE-спектр образца $\mathrm{BaSn}_{x} \mathrm{Ti}_{1-x} \mathrm{O}_{3}$ на $\mathrm{SiC}\left(\mathrm{H}^{+}\right.$, $230 \mathrm{keV})$.

\section{PIXE-спектры изолирующих образцов}

При исследовании кристаллов и пленок на подложках, являющихся хорошими изоляторами (далее - „изолирующие образцы“), наблюдается сильное влияние излучения, обусловленного падающим на образец потоком электронов, ускоренных индуцированным протонным пучком поверхностным потенциалом, который может достигать десятков kV. Как отмечалось выше, такие электроны не только производят непрерывный по энергии фон, но и создают вакансии во внутренних оболочках атомов, что приводит к генерации характеристических линий. В диапазоне $\mathrm{MeV}$-энергий зондирующего пучка сечения ионизации протонами и этими электронами соизмеримы, в области же средних энергий спектр практически полностью определяется электронной ионизацией (рис. 4).

На рис. 5 показан спектр такой же, как и на рис. 3, пленки, но на изолирующей подложке $\mathrm{Al}_{2} \mathrm{O}_{3}$ (сапфир). 


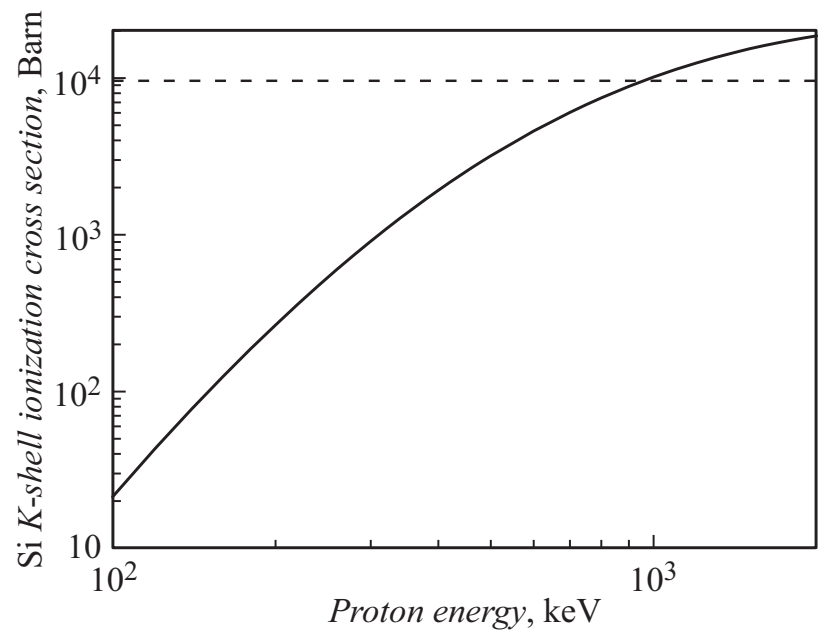

Рис. 4. Сечение ионизации $K$-оболочки кремния протонами (сплошная кривая). Штриховая линия - сечение ионизации той же оболочки электронами с энергией $8 \mathrm{keV}$. Данные взяты из работ [9] и [10].

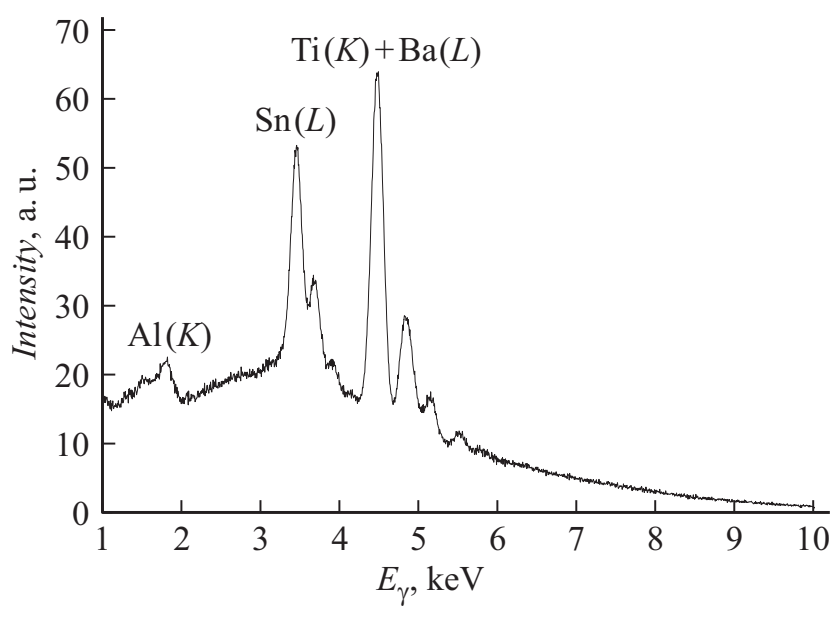

Pис. 5. РIXE-спектр образца $\mathrm{BaSn}_{x} \mathrm{Ti}_{1-x} \mathrm{O} 3$ на $\mathrm{Al}_{2} \mathrm{O}_{3}\left(\mathrm{H}^{+}\right.$, $230 \mathrm{keV})$.

Для оценки величины поверхностного потенциала нами было сделано измерение RBS-спектров обоих образцов (рис. 3,5$)$ на ионах $\mathrm{N}^{+}$с энергией $230 \mathrm{keV}$ при токах пучка, близких к токам зондирующего протонного пучка при PIXE-измерениях (регистрация обратнорассеянных ионов осуществлялась сферическим электростатическим анализатором), и проведено их моделирование (рис. 6), что позволило определить величину потенциала, составившую в данном случае $10.5 \mathrm{kV}$. Как видно из рисунка, поверхностный потенциал при RBS-измерениях влияет как на положения передних фронтов сигналов элементов образца, так и на амплитуду сигнала. Отношение энергии $E$, соответствующей переднему фронту RBSспектра при обратном рассеянии ионов с массой $m$ и энергией $E_{0}$ от элементов с массой $M$ на угол $\alpha$, к $E_{0}$, при отсутствии потенциала зарядки поверхности есть $E / E_{0}=K$, а при наличии поверхностного потенциала $\varphi-E / E_{0}=K+e \varphi(1-K) / E_{0}$, где $K(m / M, \alpha)-$ кинематический фактор, $e-$ элементарный заряд. Амплитуда RBS-сигнала от приповерхностных слоев $\propto\left(E_{0}-e \varphi\right)^{-2}$. Таким образом, влияние поверхностного потенциала на RBS-спектр возрастает с понижением энергии пучка и увеличением массы иона (уменьшением кинематического фактора), поэтому RBS-измерения на средних энергиях с использованием более тяжелых, чем $\mathrm{H}^{+}$и $\mathrm{He}^{+}$, ионов, позволяют с высокой точностью измерить поверхностный потенциал. Подробнее о применении ионных пучков средних энергий для RBSдиагностики см. [11].

Может показаться, что представленный на рис. 5 спектр даже предпочтительнее того, что показан на рис. 3: интенсивность линий возросла примерно в 100 раз, соответственно улучшилось отношение сигнал/шум, но на самом деле подобные спектры малопригодны для практических и, особенно, для научных целей. Поверхностный потенциал нестабилен, на него влияет состояние поверхности образца (наличие адсорбированных веществ), кроме того, возможны пробои и т. п., поэтому от него желательно избавляться. Существуют, помимо очевидных (нанесение проводящей пленки на поверхность образца), и более сложные способы решения задачи нейтрализации поверхностного потенциала. Например, авторами [12] было проведено исследование возможности решения поставленной задачи путем размещения вблизи поверхности образца термоэмиссионного катода, и показано, что по достижении определенной пороговой мощности нагрева катода действительно происходит нейтрализация поверхностного заряда потоком электронов с катода, о чем они судили по росту сателлита PIXE-линии, обусловленному многократной ионизацией атомов мишени, вызываемой в основном протонным ударом.

Нами предлагается альтернативный способ полного устранения поверхностного потенциала изолирующих

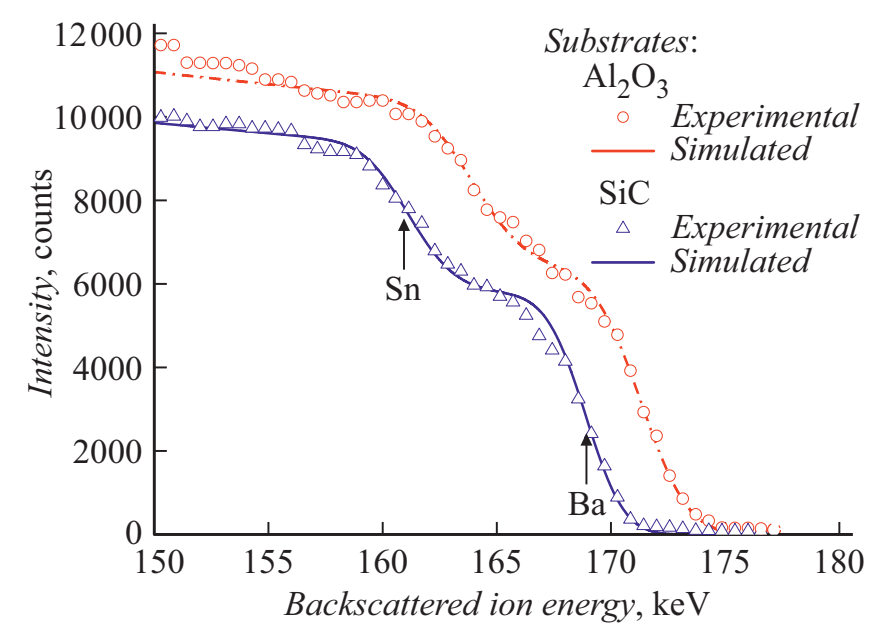

Рис. 6. RBS-спектры образца $\mathrm{BaSn}_{x} \mathrm{Ti}_{1-x} \mathrm{O}_{3}$ на $\mathrm{Al}_{2} \mathrm{O}_{3}$ и на $\mathrm{SiC}$ $\left(\mathrm{N}^{+}, 230 \mathrm{keV}\right)$. 


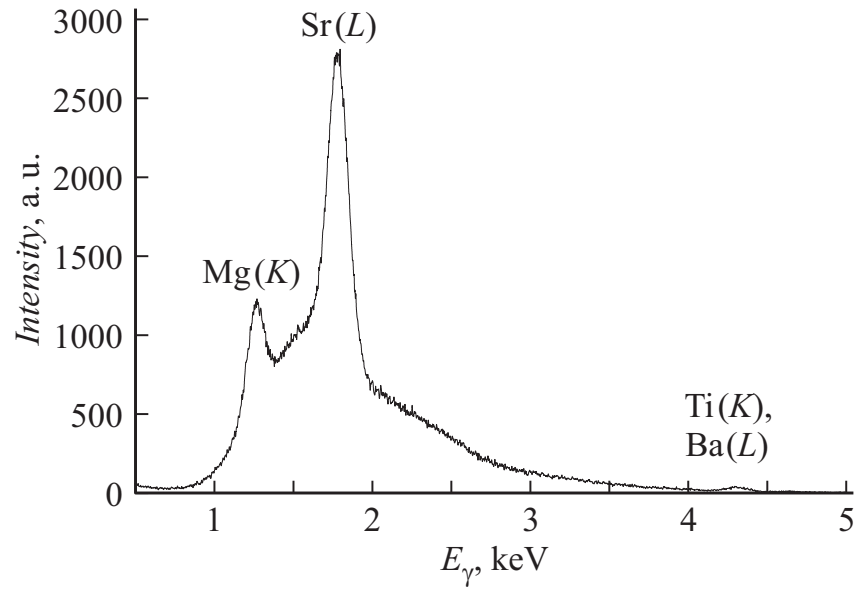

Рис. 7. РIXE-спектр образца $\mathrm{Ba}_{0.75} \mathrm{Sr}_{0.25} \mathrm{TiO}_{3}+\mathrm{Mg}$ на $\mathrm{Al}_{2} \mathrm{O}_{3}$ $\left(\mathrm{H}^{+}, 230 \mathrm{keV}\right)$.

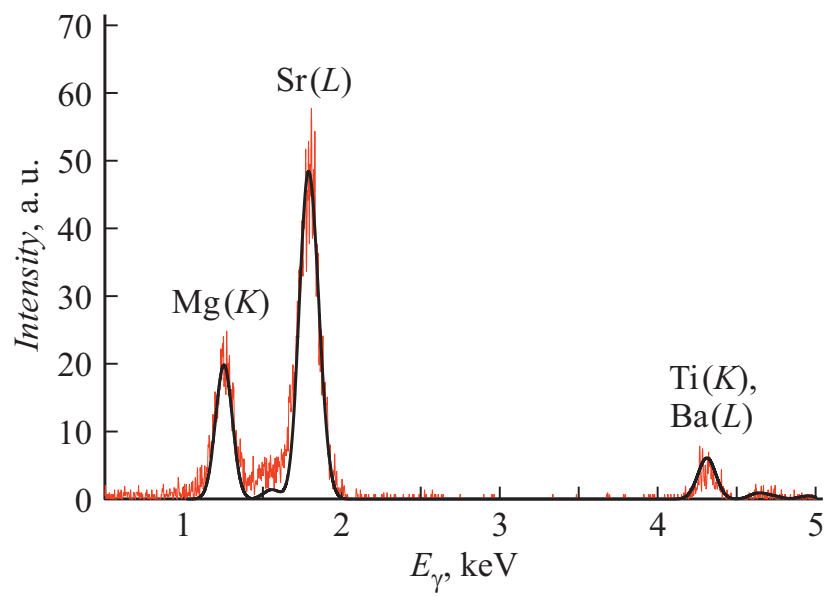

Рис. 8. PIXE-спектр образца $\mathrm{Ba}_{0.75} \mathrm{Sr}_{0.25} \mathrm{TiO}_{3}+\mathrm{Mg}$ на $\mathrm{Al}_{2} \mathrm{O}_{3}$ $\left(\mathrm{H}^{0}, 230 \mathrm{keV}\right)$. Шумящая (красная) (в on-line версии) кривая эксперимент, гладкая (черная) кривая - расчет.

образцов, а именно использование не заряженных ионных, а нейтральных атомных пучков. И если нейтрализация пучка в $\mathrm{MeV}$-диапазоне энергий представляет определенные технические трудности, то в области средних энергий задача решается сравнительно просто. На пути ионного пучка нами была установлена камера нейтрализации длиной $18 \mathrm{~cm}$ с цилиндрическими перепадниками, в которой поддерживалось давление $\mathrm{N}_{2}$ (воздуха) на уровне $(3-5) \cdot 10^{-3}$ Torr. Наличие перепадников и специальная откачка окружающего камеру нейтрализации пространства практически исключали влияние вытекающего из нее газа на давление в измерительной камере. Таким способом удавалось получить выход нейтральной компоненты на уровне $2-3 \%$ интенсивности исходного ионного пучка. Выходящий из камеры пучок очищался от заряженных компонент системой плоскопараллельных отклоняющих пластин. Отметим, что при переходе от $200 \mathrm{keV}$ к $2 \mathrm{MeV}$ сечение нейтрализации $\mathrm{H}^{+}$на $\mathrm{N}_{2}$ уменьшается примерно в 3000 раз [13].

Эффективность использования нейтральных пучков для PIXE-измерений нами тестировалась на пленке $\mathrm{Ba}_{0.75} \mathrm{Sr}_{0.25} \mathrm{TiO}_{3}$ с введенной примесью $\mathrm{Mg}$ толщиной $\sim 1 \mu \mathrm{m}$ на подложке $\mathrm{Al}_{2} \mathrm{O}_{3}$ (поликор). На рис. 7 показан PIXE-спектр, полученный на пучке протонов с энергией $230 \mathrm{keV}$. Как и на рис. 5, здесь отчетливо виден непрерывный по энергии фон тормозного излучения электронов, а также аномально большие (по отношению к ожидаемым, рассчитанным для протонного пучка) линии характеристического излучения.

Спектр того же образца, полученный на нейтральном пучке, показан на рис. 8 .

Там же приведен результат расчета, выполненного для протонного пучка с энергией $230 \mathrm{keV}$ при отсутствии поверхностного потенциала. Данные по сечениям ионизации внутренних оболочек, сечениям торможения, квантовым выходам, коэффициентам Костера-Кронига и коэффициентам поглощения взяты из работ $[9,14-17]$. Моделирование позволило измерить отношение концентраций $\mathrm{Mg}$ и $\mathrm{Sr}$, составившее в этом случае 0.48 . Несмотря на резкое падение интенсивности и отношения сигнал/шум, именно такие „очищенные“ от посторонних сигналов спектры позволяют надежно установить состав исследуемых образцов.

\section{Заключение}

Представленные результаты показывают, что использование ионных пучков средних энергий для твердотельной диагностики методом PIXE является эффективным инструментом при исследовании как кристаллов, так и тонких (до $1 \mu \mathrm{m})$ слоев и пленок, в том числе являющихся сильными изоляторами, поскольку позволяет сравнительно легко решать проблемы, связанные с исключением тормозного и характеристического излучений, обусловленных движением электронов в исследуемом образце.

\section{Финансирование}

Работа выполнена по Государственному заданию, тема № 0040-2019-0023.

\section{Конфликт интересов}

Авторы заявляют, что у них нет конфликта интересов.

\section{Список литературы}

[1] K. Ishii. Quantum Beam Sci., 3 (2), 12 (2019). DOI: https://doi.org/10.3390/qubs3020012

[2] R.S. Bhattacharya, P.P. Pronko. Appl. Phys. Lett., 40 (10), 890 (1982). DOI: https://doi.org/10.1063/1.92938

[3] J.R. Bird, J.S. Williams. (Eds.). Ion Beams for Material Analysis (Academic Press, Sidney, 1989). 
[4] K. Ishii, H. Yamazaki, S. Matsiyama, W. Galster, T. Satoh, M. Bugnar. X-Ray Spectrom. 34, 363 (2005). DOI: https://doi.org/10.1002/xrs.838

[5] C.K. Li, K.W. Wenzel, R.D. Petrasso, D.H. Lo, J.W. Coleman, J.R. Lierzer, E. Hsieh, T. Bernat. Rev. Sci. Instrum., 63 (10), 4843 (1992). DOI: https://doi.org/10.1063/1.1143578

[6] J. Miranda. Nucl. Instr. Meth. B, 118, 346 (1996). DOI: https://doi.org/10.1016/0168-583X(95)01176-5

[7] L.C. Feldman, J.W. Mayer, S.T. Picraux. Material Analysis by Ion Channeling (Academic Press, NY., 1982).

[8] K.H. Ecker, Z. Quan, T. Schurig, H.P. Weise. Nucl. Instr. Meth. B, 118, 382 (1996).

DOI: https://doi.org/10.1016/0168-583X(96)00249-2

[9] D.D. Cohen, M. Harrigan. Atomic Data and Nuclear Data Tables., 33 (2), 255 (1985).

DOI: https://doi.org/10.1016/0092-640X(85)90004-X

[10] X. Long, M. Liu, F. Ho, X. Peng. Atomic Data and Nuclear Data Tables., 45 (2), 353 (1990).

DOI: https://doi.org/10.1016/0092-640X(90)90011-8

[11] V.V. Afrosimov, R.N. Il'in, V.I. Sakharov, I.T. Serenkov. ФТП, 41 (4), 497 (2007). [V.V. Afrosimov, R.N Il'in., V.I. Sakharov, I.T. Serenkov. Semiconductors, 41 (4), 487 (2007). DOI: http://dx.doi.org/10.1134/S1063782607040252]

[12] H. Yamanaka, K. Hasegawa, K. Maeda. Nucl. Instr. Meth. B, 109/110, 203 (1996).

DOI: https://doi.org/10.1016/0168-585X(95)00906-X

[13] B. Navinšek. (editor). Physics of Ionized Gases (Hercegnovi, Yugoslavia, 1970) p. 69.

[14] J.F. Janni. Atomic Data and Nuclear Data Tables., 27 (4-5), 341 (1982).

DOI: https://doi.org/10.1016/0092-640X(82)90005-5

[15] M.O. Krause. J. Phys. Chem. Ref. Data, 8, 307 (1979). DOI: https://doi.org/10.1063/1.555594

[16] J.L. Campbell. Atomic Data and Nuclear Data Tables., 85 (2) 291 (2003). DOI: https://doi.org/10.1016/S0092-640X(03)00059-7

[17] B.L. Henke, E.M. Gullikson, J.C. Davis. Atomic Data and Nuclear Data Tables., 54 (2), 181 (1993). DOI: https://doi.org/10.1006/adnd.1993.1013 